\title{
Arc Initiation for the Electromagnetic Powder Deposition Gun
}

By:

R.L. Sledge

J.L. Bacon

D.G. Davis

R.J. Polizzi

J.R. Uglum

R.C. Zowarka

United Thermal Spray Conference, Sept. 15-18, 1997, Indianapolis Indiana

PR - 229

Center for Electromechanics

The University of Texas at Austin

PRC, Mail Code R7000

Austin, TX 78712

(512) 471-4496 


\title{
Arc Initiation for the Electromagnetic Powder Deposition Gun
}

\author{
R.L. Sledge, J.L. Bacon, D.G. Davis, R.J. Polizzi, J.R. Uglum, and R.C. Zowarka \\ Center for Electromechanics \\ The University of Texas at Austin \\ Austin, Texas
}

\begin{abstract}
The Electromagnetic Powder Deposition (EPD) process converts pulsed electrical energy into kinetic and thermal energy to accelerate and heat powder material to conditions suitable for bonding. A high pressure plasma armature is electromagnetically accelerated using a railgun. A supersonic pressure wave is created when the armature accelerates through and "snowplows" the ambient gas ahead of it. The gas column is heated, compressed, and accelerated to the entrainment section of the gun, where some of the thermal and kinetic energy is transferred to an injected stream of powder material. The acceleration burst is repeated rapidly to supply the required deposition rate and to achieve steady thermal conditions.
\end{abstract}

Development of a starter plasma which is reliable at ambient pressure was a major programmatic task. Generation of a low pressure linear arc required to form a planar armature during the pulsed event was investigated. Several geometries (pointto-point breakdown, rail-to-rail breakdown, and confined glow discharge) were explored using different voltage sources (dc, $60 \mathrm{~Hz}$ ac, $150 \mathrm{MHz}$ rf, pulsed). Satisfactory operation of the confined glow discharge approach at atmospheric pressure was achieved using rf excitation. Results of testing under the various scenarios are presented and critiqued.

THE GOAL OF THE EPD PROGRAM is to develop coating and build-up processes which exceed the performance of existing technologies. Improvements are expected in the areas of materials compatibility, bond strength, coating strength and density. A plasma armature railgun technique [1] studied two decades ago has been selected for accelerating powder material to velocities in excess of those achieved by existing thermal spray processes. The railgun consists of two metallic rails with insulating sidewalls separating them (Figure 1). A low mass plasma armature, initiated in the breech, is used to commute the gun current between the rails.
In this configuration, current flow generates a magnetic field within the bore, behind the armature. The current carrying ions and electrons of the plasma armature are acted on by the magnetic field, accelerating the armature forward. As it is accelerated, it collisionally interacts with the ambient gas in the bore and accelerates the gas to the same velocity. This mechanism of momentum transfer is referred to as the snowplow. Essentially, the arc armature acts like a piston, pushing the gas in front of it as it moves down the gun bore. The gas eventually passes the powder injection point, exerting viscous drag and accelerating the powder particles to a velocity of 2 $\mathrm{km} / \mathrm{s}$. High velocity is the most important attribute of electromagnetic powder deposition (EPD) as a coating process. Another unusual aspect of EPD, shared with some detonation processes, is the repetitive pulse mode of operation $(\sim 30 \mathrm{~Hz})$. While substrate heating is reduced by thermal relaxation between pulses, some heating of the powder will occur via interaction with the plasma and the gas column heated by a shock front propagated through the gas. Since EPD will be used at atmospheric pressures, oxidation of deposition surfaces may occur. To avoid oxidation, the process will employ an inert gas, argon, for the purpose of substrate shielding as well as for plasma seeding and powder feeding.

To behave as a piston, the armature must fill the entire transverse section of the bore. For maximum efficiency, the ideal shape of the armature would be a plane or "sheet". A major challenge of the process, and the focus of this paper, is the initiation of such an armature. Historically, plasma railguns have been used to accelerate gases. Unlike the EPD process, however, the plasma initiator took the form of an exploding fuse. The pulse power sources driving these wire or foil fuses operated with risetimes of only a few microseconds so that the fuse material would be thoroughly vaporized. In the EPD process, because a longer acceleration pulse is needed, the risetime is also considerably longer due to the characteristics of the power supply. The power source will provide a $20 \mu$ s ramp-up, after which a constant $160 \mathrm{kA}$ current is delivered for $100 \mu \mathrm{s}$. Another important reason for 


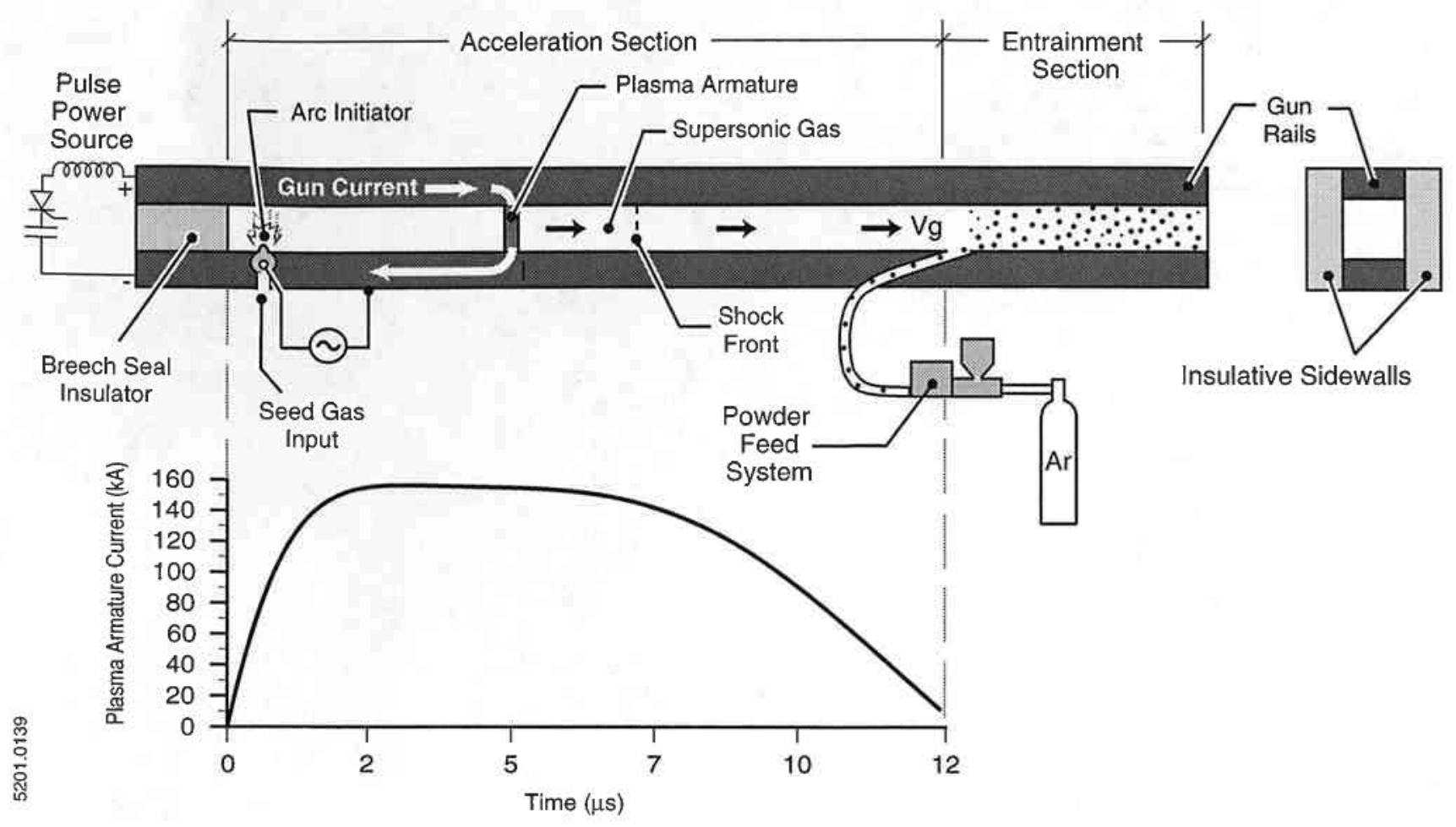

Figure 1 - EM powder deposition gun

eliminating the fuse-type initiator is that the products of exploded fuses would be detrimental to the purity of the coatings. The possibility of using a fuse material which is identical to the deposition material was considered but was determined to be impractical due to material availability. It would be prohibitively expensive to develop a conductive solid form of all feed alloys and mixtures, some of which are available only in powder form. Moreover, the necessity of fuse reloading between every shot would introduce a great deal of risk at the planned rate of repetition. With these constraints, fuses were omitted as an option for developing the EPD process, and a new way of initiating the plasma was needed.

Early in the program, questions regarding the environmental conditions of the process were unanswered. The feasibility of operating at atmospheric pressure was under investigation, with arc initiation being one of the major issues. Common applications such as neon and fluorescent lighting were assurance that an inert gas could be excited to a glow discharge plasma state under low pressure conditions. A fundamental experiment was executed using simple, rounded electrodes in a vacuum chamber backfilled with argon. Direct current and $60 \mathrm{~Hz}$ supplies for plasma generation were selected because they were readily available and produced low radiated noise. The electrodes were tested at increasing pressure, but filamentation was observed before atmospheric pressure was reached. Several electrode schemes, designed to create a line source plasma that could evolve into a sheet cur- rent, were developed in concept but not all tested due to the rapid progress of one of the schemes. Not achieving success with the conventional power sources, the research moved to an rf power supply in the megahertz regime. The scheme that provided the most favorable results utilized the rf power source. For protection of the rf source, the starter arc could not bridge both rails. In addition, the electrode had to provide a line of plasma which would initiate the planar arc required for snowplow. The geometry that satisfied this requirement was a coaxial design featuring a cavity inset directly into the low potential gun rail. This design was evolved in a set of carefully planned experiments and then integrated into the first generation research accelerator known as the Bench Test Railgun. The snowplow arc was successfully demonstrated using this design.

\section{Plasma Instability}

To operate in the snowplow mode, the railgun armature must maintain a planar form which fills the bore cross-section during the entire acceleration event. Because the armature is a gaseous plasma, instabilities could potentially be a major difficulty. Two specific instability mechanisms known to be important in other plasma arc applications were studied for the plasma armature process.

The first mechanism considered is the filamentation, or thermal runaway, instability. It arises physically from two 
phenomena. First, parallel currents attract each other, so the arc current flow wants to pinch naturally. Second, the resistivity of a plasma falls as the plasma temperature increases (Spitzer resistivity). The analysis reveals in this particular case that the pinch effect is balanced by internal gas pressure and is not a major stability issue. The analysis focused on the thermal runaway aspect of the problem. For normal heat conduction, thermal conductivity has a weak temperature dependence. For dense plasmas of interest here, however, heat conduction is by black body radiation and the effective conductivity scales roughly as the third power of temperature. When these scalings are used in a stability equation derived for the EPD process [2], the analysis shows that stability will occur. Once established, the planar armature of the EPD railgun accelerates intact and the filamentation instability is not a problem.

The second mechanism studied is the Rayleigh-Taylor instability. The Rayleigh-Taylor instability is a generic problem which occurs when a light fluid (here magnetic field) is used to accelerate (or support) a heavier fluid (here plasma). A good example of this instability is the attempt to support mercury on top of water in a container. This physical state can be realized, but common experience shows that any small perturbation to this system quickly leads to a state with the mercury on the bottom and the water on top. As applied to the EPD process, the concern is that the magnetic field would try to slip past the plasma, leaving plasma filaments behind. Physically, however, the magnetic field cannot rush past the current which is generating it, but must diffuse through the conducting medium. A detailed analysis of the growth rate has shown that when established, the planar arc will have a low instability growth rate and will experience small change in geometry during the acceleration event. That is, once the planar arc is established, the rate of the armature deforming to an unstable shape is low and the Rayleigh-Taylor instability is not expected to be a problem.

\section{Glow Discharge Testing}

After determining that a stable snowplowing plasma armature can be realized, the method of forming the initial plasma was addressed. The feasibility of operating at atmospheric pressure was investigated, with arc initiation being one of the major tasks. Lessons learned would be applied to the design of electrodes and power supply for the arc initiator portion of the Bench Test Railgun. The goal of the testing was to determine if and at what pressure a sustainable, diffuse three-dimensional plasma (glow discharge) generated between two cylindrical electrodes would pinch to a planar sheet arc or would transition to a single filamentary arc or multiple filamentary arcs. The bottom end pressure, about 50 mTorr, was the starting point for this test because it was known to produce glow discharge. The upper limit of the test, 1 atmosphere, is the pressure considered by industry to be the most convenient for thermal spray processes.

The plan was to place two electrodes in an argon backfilled vacuum chamber, apply voltage to ionize the rarefied argon gas between the electrodes, and observe plasma characteristics while raising the chamber pressure. To minimize the effects of electrode shape, cylindrical electrodes specified by ASTM 149 were used (Figure 2a). Two power sources were used. A current limited ac power supply (neon light transformer) was operated at $60 \mathrm{~Hz}$. This transformer will produce an open-circuit voltage of $7500 \mathrm{~V}$ and will current limit at $20 \mathrm{~mA}$. A dc power supply was also used to observe polarity effects and to more easily determine the electrical resistance of the plasma. To compare the dc power supply to the ac process, the dc was set at $20 \mathrm{~mA}$.

Testing with the ac power supply revealed glow discharge around both electrodes at low pressures (Figure 2b). In the dc mode, glow discharge was observed around the cathode only. Other than the glow being centered differently between the ac and dc modes, the transitional range and other
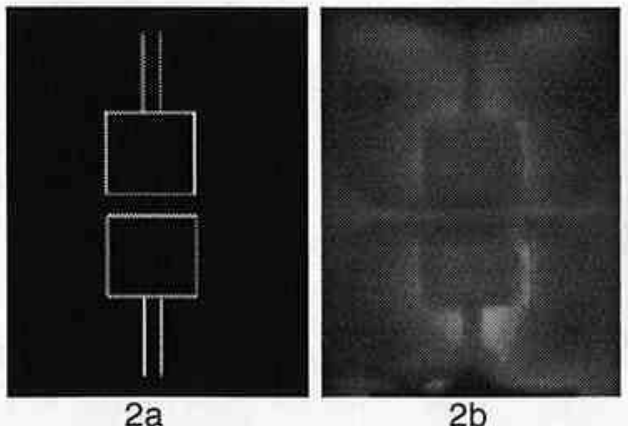

Test

Electrodes

ASTM 149
$2 b$

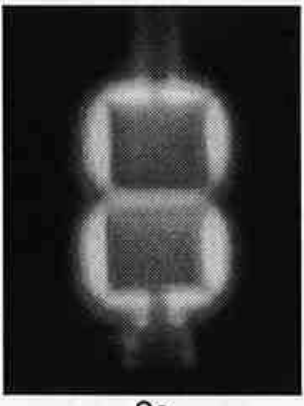

Encompassing Glow Discharge 2c

2 Torr

Reduced

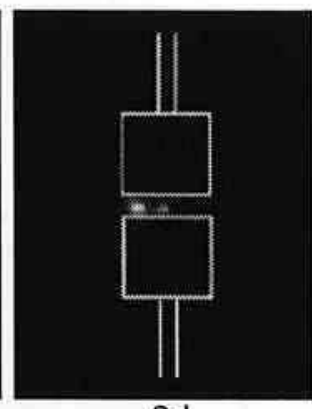

2d

70 Torr

Multiple

Glow Discharge
Filamentary Arcs

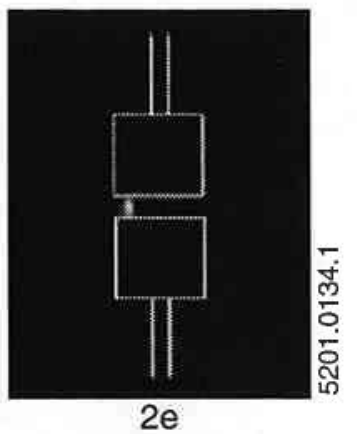

760 Torr

(1 atm)

Single Filament

Figure 2 - Glow discharge test 
plasma observations were similar for both setups. As pressure was increased from $10 \mathrm{~m}$ Torr to 1 Torr, the violet colored glow was observed to move closer to the electrodes and increase in intensity. At about 1 Torr, the glow was seen mostly in the $4 \mathrm{~mm}$ gap between the electrodes.

While the plasma did not form into a sheet, a range of pressure where the plasma transitioned from a glow discharge to filamentation arcs was found. Up to about 10 Torr, the violet colored glow was seen full-width between the electrodes, but was not pinched to a small axial thickness (Figure 2c). Between 10 and 50 Torr, with some glow still present in the gap, sparks were observed and regions of higher intensity light were seen. As pressure increased to 70 Torr, the intense areas appeared to condense further and become more dynamic, eventually constricting into multiple filamentary arcs, rapidly traversing the gap width (Figure 2d). In the region of $1 \mathrm{~atm}$, only a single arc was present, ultimately settling at one fixed location (Figure 2e).

Through glow discharge testing, the difficulty of creating a planar plasma at $1 \mathrm{~atm}$ was recognized and possible solutions conceptualized. Different power sources and electrode geometries were considered. Three concepts were eventually pursued.

\section{Ignition System: A Point-to-Point Breakdown}

In terms of operation and geometry, the ignition system concept was one of the more complex concepts proposed. One reason for considering this option was isolation of the arc initiation circuit from the main discharge circuit. In this design, two pointed electrodes are installed through the insulative sidewalls of the railgun (Figure 3). The circuit would generate a linear arc between the points which, because of its orientation across the bore, could evolve into a sheet arc between the rails upon discharge. Although both electrodes are isolated from galvanic connection, an electrical coupling through plasma contact is possible, implying a certain degree of risk for this concept. A second reason for considering this option was the possibility of using as the power source an inexpensive automotive ignition system.

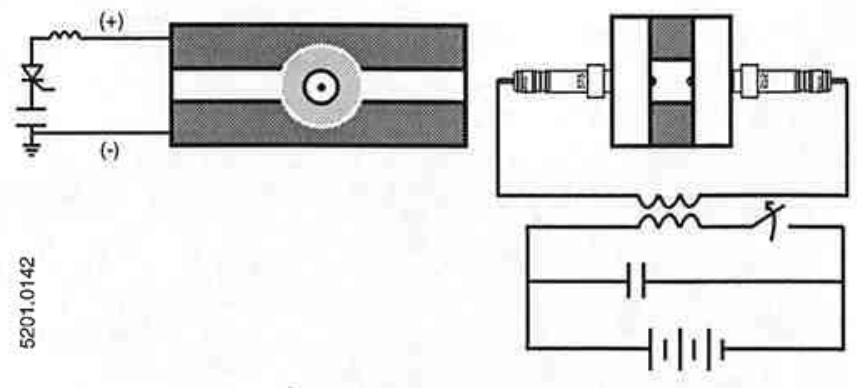

Figure 3 - Isolated point-to-point electrodes
The ignition system concept, however, would require accurate timing with the discharge circuit because of its pulsed mode of operation. The accurate timing requirements would create a significant risk and difficulty for the EPD process.

While the point-to-point geometry was never tested in a railgun event, a mockup was constructed and tested using the $60 \mathrm{~Hz}$ ac power supply, under conditions similar to those of the glow discharge experiments. The mockup was constructed from the same type of square $(1.27 \mathrm{~cm})$ copper bar intended for construction of the Bench Test Railgun. Like the glow discharge tests, the opposing point geometry produced a wide glow discharge at pressures below 10 Torr, transitioning to filaments at 100 Torr. During this testing, a second method was developed which showed greater promise. The rapid success of the second method, presented in the next section, led to the abandonment of the ignition system concept.

\section{Coaxial Cavity Electrode: A Confined Glow Discharge}

The coaxial cavity electrode concept used a high frequency alternating current to establish a confined glow discharge in a controlled region of one rail. This concept employed a power source secondary to the main capacitive discharge source, so there were risks associated with the interaction of both sources. The configuration is essentially a three terminal device, with no means of fully isolating the two power sources. One concern was that the impedance of the secondary source would be inadequate to protect it from a destructive internal current spike. Another concern was that of electromagnetic noise interference with diagnostic and control instruments.

The cavity geometry is essentially a coaxial assembly, with the internal conductor being a solid wire electrode connected to a high frequency source (Figure 4). The outer conductor is defined by the cavity, a hole drilled through the rail such that a narrow portion of the hole breaks out into the bore region creating a uniform slot spanning the full bore width. An air gap exists between the electrode and the cavity wall where argon is injected for ionization. The argon injection inlets are four small holes entering normal to the cavity wall

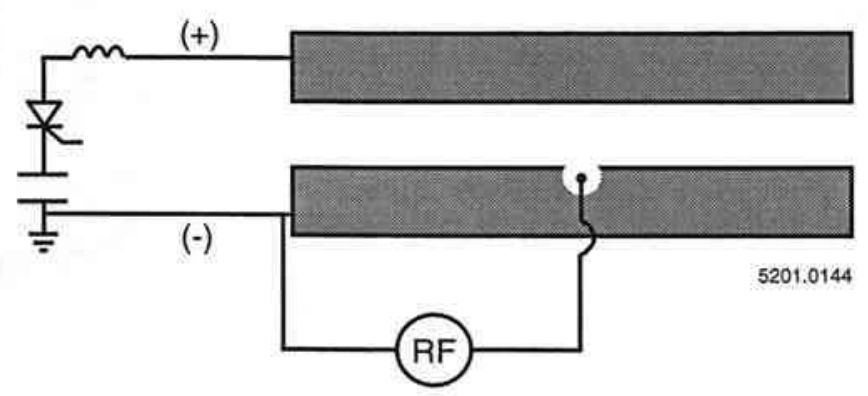

Figure 4 - Cavity electrode 
on a line directly opposite the rail slot. In principle, a line of plasma protruding from the slot would act as an ideal armature initiator, providing both a full-width voltage stressor and a starter quantity of conductive gas.

Cavity diameter was 2.7 multiplied by the electrode diameter, a proportion for minimizing voltage stress. A cavity test mockup included two cavity sizes, approximately 7 $\mathrm{mm}$ and $3 \mathrm{~mm}$ in diameter. At this stage, the mockup cavities were simple drilled holes with no breakout slot and no argon injection inlets.

The $7 \mathrm{~mm}$ cavity was first tested using the ac power supply. Results were similar to those seen in glow discharge tests, i.e., as pressure approached $1 \mathrm{~atm}$, plasmas were observed to pinch to filament arcs. The desired full-width arc was not demonstrated, so plans for high frequency testing proceeded. An rf power amplifier was specified to produce the voltage and wattage required to strike and sustain the arc. The possibility of designing an inexpensive prototype amplifier was considered, but programmatic constraints dictated the selection of a commercially available unit. A unit which roughly fit the specifications was located. The unit was an ENI model A-300 with the following specifications: $300 \mathrm{kHz}$ - $35 \mathrm{MHz}, 300 \mathrm{~W}, 55 \mathrm{dBm}$ gain, $200 \mathrm{~V}$ peak.

The voltage capability of this unit was lower than desired, prompting a concern about the ability to initiate a voltage breakdown in the cavity. The $7 \mathrm{~mm}$ cavity was first tested at about $2 \mathrm{MHz}$, the upper limit of the available signal generator. Voltage breakdown of the cavity occurred intermittently but could not be accomplished reliably, even at pressures down to $2 \mathrm{mTorr}$. One modification of the system was made. The copper electrode wire was replaced with a pure tungsten wire, resulting in a noticeable improvement in electrode durability. The copper wire would bend or melt if subjected to continuous duty. The tungsten under similar conditions did not exhibit any signs of heat distress except for some surface discoloration. Specific behavior of the $2 \mathrm{MHz}$ plasma in the argon backfilled vacuum was noted. First, breakdown could be initiated by cracking the backfill valve, presumably creating a pressure disturbance at the cavity. Second, the arc would extinguish when pressure reached about 185 Torr. Also, the plasma, although spanning the full rail width, exhibited multiple $(>10)$ filamentary concentrations rapidly moving within the glow discharge.

Because an even glow discharge was sought, and because initiation of the arc was unreliable, an effort was made to operate the test equipment at higher frequencies. A $40 \mathrm{MHz}$ signal generator was acquired and testing resumed at higher frequencies. An observation was made that, for the given test conditions, breakdown could be initiated more reliably as oscillator frequency increased. Tests were ultimately performed at $35 \mathrm{MHz}$, the upper limit of the amplifier. At this frequency, the plasma could be initiated at higher pressures and the plasma was a glow discharge which was even and free of filament arcs. Also, the plasma sustained itself to higher pressure, up to 600 Torr, approaching the target pressure of 760 Torr $(1 \mathrm{~atm})$.
The $3 \mathrm{~mm}$ cavity mockup was tested. Due to the small scale, it was expected to initiate the arc and sustain it at higher pressures. This hypothesis was based on the inverse relationship between vacuum gap geometry and the pressure at which voltage breakdown occurs. Indeed, after initiating a breakdown at a low pressure, the plasma was sustained all the way to $1 \mathrm{~atm}$. With the target pressure reached, the cavity test was taken to the next phase. The mockup rail was machined such that the rail wall intersected the cavity. The cavity then opened into the gun bore region by way of a full width slot, with an opening approximately $1 / 3$ the width of the cavity diameter. The open cavity was tested and found to perform as well as the closed cavity, although neither version would initiate an arc at $1 \mathrm{~atm}$ using the available power source. A new rf amplifier and signal generator were acquired for higher frequency testing. The new amplifier, an ENI model 3200L, has a higher frequency range but a lower power rating, as follows: $120 \mathrm{kHz}-150 \mathrm{MHz}, 175 \mathrm{~W}, 55 \mathrm{dBm}$ gain, $200 \mathrm{~V}$ peak.

During testing of the new oscillator system, it was again observed that higher frequencies would allow arc initiation at higher pressures. In fact, by driving the system to peak frequency, $150 \mathrm{MHz}$, the plasma could be initiated and sustained (in the $3 \mathrm{~mm}$ cavity) at $1 \mathrm{~atm}$. With this milestone, testing of this mockup was completed and the cavity electrode was integrated into the Bench Test Railgun.

\section{Rail Voltage Stressors: A Rail-to-Rail Breakdown}

While the rf-cavity initiator appeared to be a viable solution, another proposal was suggested which held enough advantages to justify testing. The rail voltage stressor concept would depend on the voltage rise of the primary current pulse to initiate the arc, with the site of initiation determined by a voltage stressor. The concept was that a filamentary arc might expand enough to create a full-width armature. This method would simplify the system by removing the rf power source and the third terminal. While two configurations were suggested, only one was tested. The first method was not tested, but featured opposing rounded risers on the rail surfaces (Figure 5). While these voltage stressors were planned to be full rail width, there was no certainty that the arc would be centered in the bore. In fact, it was considered more likely that the breakdown would occur at the edges of the bore where the higher field stresses exist.

The second configuration featured a stress riser pin centered on one rail only (Figure 6). The advantage of the single pin is its positional flexibility, i.e., its length can be easily extended. Because this initiation method is dependent on the main discharge, it was tested as part of the BTR experiments and is reported in the following section.

\section{Arc Initiation in the Bench Test Railgun}

In order to evaluate the snowplow process, the position of the plasma armature through time must be known. Axial b-dot probes, which convert magnetic flux to voltage signals, 


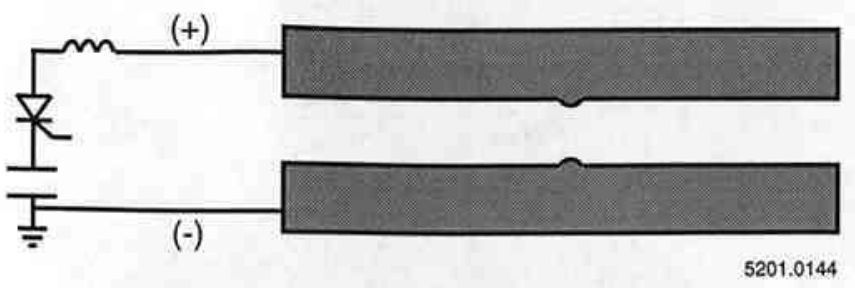

Figure 5 - Edge-to-edge voltage stressors

are used with railguns for the purpose of recording the passing time of the armature [3]. Fiber optic techniques can also be used to verify the position of the armature, which emits light in the ultraviolet range. Comparing these measured positions to simulated positions is one way to determine if the snowplow mechanism is working. A sheet armature filling the bore cross section will accelerate slower than a partial sheet or filamentary arc due to the quantity of gas being pushed. Another evaluation method is the analysis of pressure data. Dynamic (piezoelectric) pressure transducers, installed in the rail insulators, can determine when and to what extent the ambient gas is compressed. This technique provides additional insight into the stability of the armature. Separate BTR tests were conducted using the cavity electrode scheme and the stress-pin scheme. With the $3 \mathrm{~mm}$ cavity design integrated into the negative rail, the rf source was operated at $150 \mathrm{MHz}$ to excite the electrode. Operating the rf source at $30 \mathrm{~W}$ forward power with an argon flow rate of 0.23 $\mathrm{m}^{3} / \mathrm{hr}$ provided a glow discharge which protruded slightly from the cavity slit into the bore, spanning the full width of the negative rail. At this power, the rf signal did not interfere with b-dot signals, but pressure transducer readings were occasionally affected. While a lower power setting $(15 \mathrm{~W})$ was initially used, the $30 \mathrm{~W}$ value was settled upon because the plasma continued to glow following discharge. A dc voltage was applied across the rails in order to verify the breakdown properties with the cavity plasma. The dc breakdown voltage was $13 \mathrm{kV}$ in air, $4.2 \mathrm{kV}$ in argon, and $3.0 \mathrm{kV}$ with the rf-plasma.

Results of railgun testing verified the proper operation of the cavity initiator. Numerous tests demonstrated, through bdot, fiber optic, and pressure transducer data, that the snowplow mechanism occurred [2,3]. Pressure levels and armature timing agreed with simulated values. A high speed digital framing camera was also used to verify proper acceleration of the gas column. In addition to successful photography of the armature [3], several 330 micron glass beads were photographed travelling at $0.6 \mathrm{~km} / \mathrm{s}$, in agreement with the simulation code [2].

For further documentation of the cavity plasma, the high speed camera was aimed into the bore of the gun by way of a $45^{\circ}$ mirror placed at the muzzle. The camera was used to col-

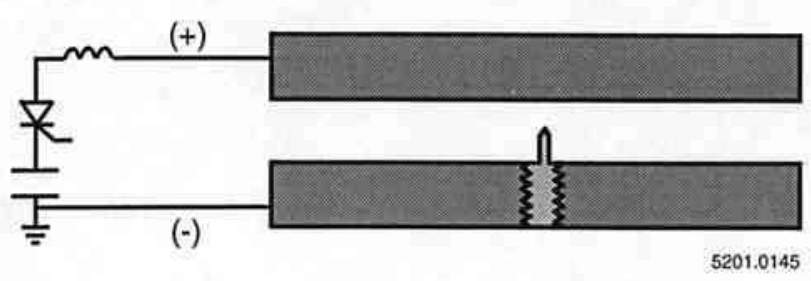

Figure 6 - Point-to-rail voltage stress pin

lect eight exposures of the arc initiator using a $10 \mathrm{~ns}$ exposure time and a 20 ns inter-frame separation. The arc's homogeneity and the region it occupied were observed. The arc was steady and did not vary between frames. It was seen as a faint line with a few beads of light which protruded less than $1 \mathrm{~mm}$ into the bore (Figure 7). Although several attempts were made to photograph the transition of the starter arc to the accelerating armature, arc visibility was poor and time constraints dictated that the camera be repositioned for acceleration testing.

BTR testing of the stress-pin method featured a $1 \mathrm{~mm}$ diameter tungsten pin installed in the negative rail. Shots were made with the pin protruding from the negative rail at lengths varying from 0.76 to $6.4 \mathrm{~mm}$. Snowplow pressures were compared between the stress-pin tests and the cavity electrode tests. Railgun shots initiated by the rf plasma developed snowplow pressures averaging $65 \%$ higher than those initiated by a voltage stress pin. Figure 8 is a typical pressure waveform for a stress pin shot, showing a peak snowplow

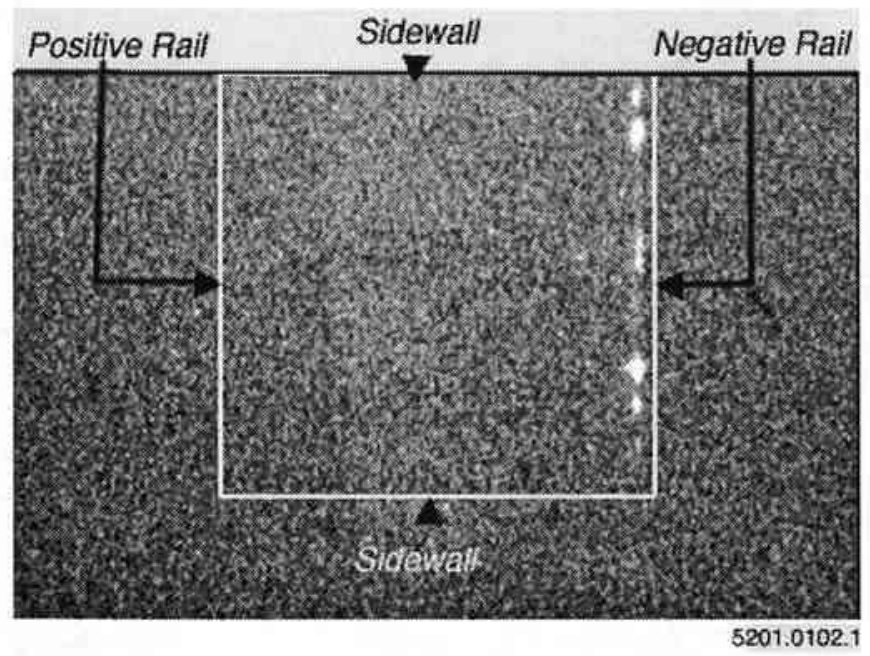

Figure 7 - View of the rf-generated plasma from the muzzle of the Bench Test Railgun 
pressure of $723 \mathrm{psi}$ at $67 \mathrm{~ms}$. The average peak pressure of six, $5 \mathrm{kV}$ shots was $590 \mathrm{psi}$. Figure 9 is a typical pressure waveform for a rf-plasma shot, showing a peak snowplow pressure of $1000 \mathrm{psi}$ at $67 \mathrm{~ms}$. The average peak pressure of six, $5 \mathrm{kV}$ shots was $977 \mathrm{psi}$.

In addition to the pressure readings, the $b$-dot and fiber optic signals also supported the hypothesis that the stress pin gun did not snowplow as efficiently as the rf initiated tests. The b-dot and fiber optic readings indicated that armature velocities for the stress pin tests were more than $25 \%$ faster. Higher velocities are generally desirable for the process. However, with other parameters equal but lower pressures measured, the higher velocities support the theory that the pin-initiated armature did not snowplow the ambient gas as effectively as the rf-generated armature. Instead, the armature accelerated past the gas without pushing it forward.

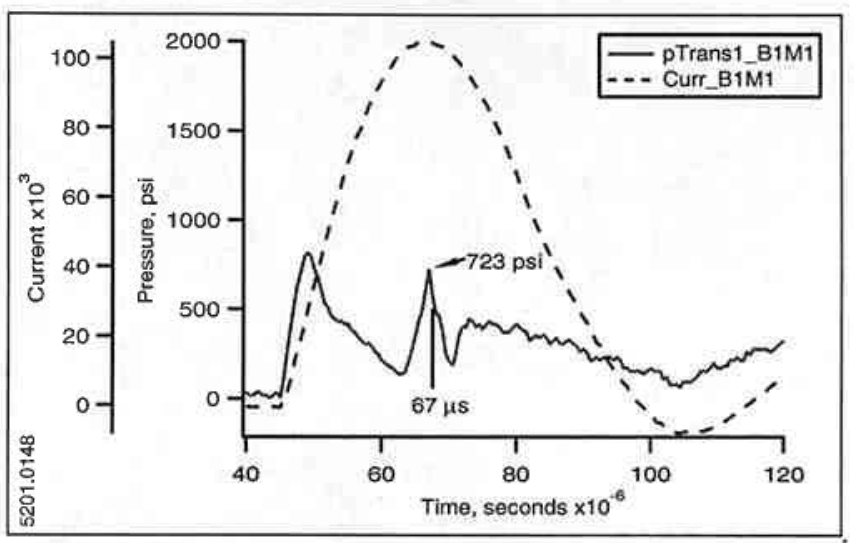

Figure 8 - Typical pressure waveform from a $5 \mathrm{kV}$ test of the Bench Test Railgun using the voltage stress pin as the arc initiator

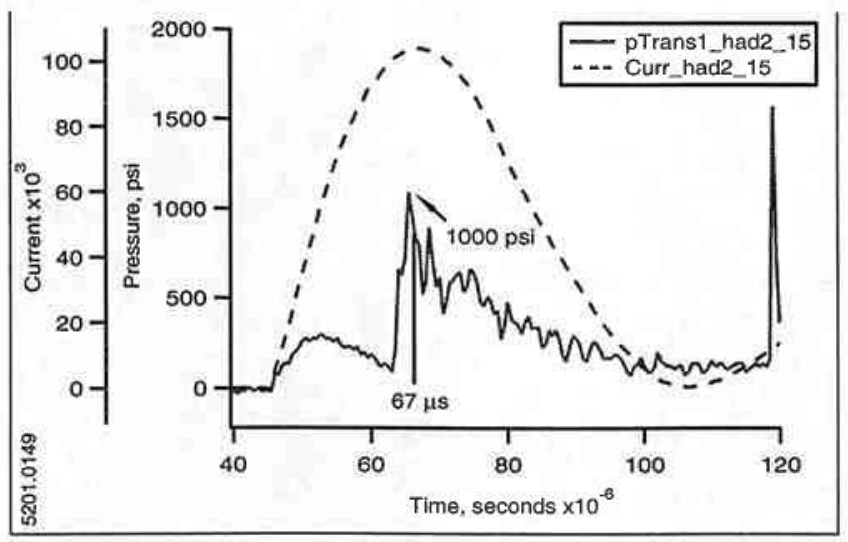

Figure 9 - Typical pressure waveform from a $5 \mathrm{kV}$ test of the Bench Test Railgun using an rf-powered plasma as the arc initiator
While the stress-pin method could simplify the EPD system by eliminating the complication of a second power circuit, the performance tradeoff was considered to be unacceptable for the process. Thus, the rf-powered cavity electrode was adopted for continued testing of the Bench Test Railgun and for integration in the next generation Square Bore Accelerator.

\section{Conclusion}

Starting a gas accelerating plasma railgun without the use of an exploding fuse was a new area of investigation. Previous research used metal foils to initiate a snowplowing arc. Thus, it was not known prior to this development whether the snowplow mechanism could be achieved without a fuse initiator, but the EPD process required that fuses be avoided for several reasons. Many deposition materials of interest are unavailable in the form required for a fuse, and the vaporization of a different material would contaminate the coating. Moreover, rapidly reloading fuses at the $30 \mathrm{~Hz}$ firing rate was considered high risk.

A process that can operate at atmospheric pressure is desired for maximum system utility. Several initiation systems were tested under vacuum conditions, raising the chamber pressure to find the threshold for sustaining glow discharge. Eventually, a $150 \mathrm{MHz}$ ac source, powering a coaxial cavity electrode at $30 \mathrm{~W}$, was determined to be a promising initiator because it created a line glow discharge at atmospheric pressure. After integrating the cavity electrode into a test railgun, the rf plasma source was proven successful in generating a planar arc that led to the successful snowplow process, confirmed by four independent experimental diagnostics, b-dots, pressure transducers, fiber optics and high speed photography.

\section{Acknowledgment}

This research was sponsored by the United States Air Force, Tinker Air Force Base at Oklahoma City, through a contract administered by ARINC Corporation.

\section{References}

[1] J. W. Shearer, et al, "Xenon Shock Waves Driven by High Magnetic Fields," Proc. 2nd International Conf. on Megagauss Magnetic Fields, 1979.

[2] J. Uglum, et al "Scaling Analysis of the Electromagnetic Powder Deposition Gun," United Thermal Spray Conference, September 1997, Indianapolis, Indiana.

[3] J. Bacon, et al, "The Diagnostic History of a New Electromagnetic Powder Deposition System," United Thermal Spray Conference, September 1997, Indianapolis, Indiana. 